\title{
Endovascular Coiling of Intracranial Aneurysms
}

\author{
Naoya Kuwayama
}

\subsection{Introduction}

Since the development of Guglielmi detachable coil (GDC), endovascular technique for coiling has become widely accepted as one of the option treatments of intracranial aneurysms.

The randomized trial of ISAT [1] proved the superiority of the aneurysm coiling than clipping for the patients with subarachnoid hemorrhage. Endovascular aneurysm treatment has been rapidly developing in technique, and its indication has been also rapidly spreading with the development of a variety of adjunctive techniques, including simple coiling, catheter- or microwireassisted coiling, double-catheter technique, balloon remodeling technique, and stent-assisted technique. In this educational chapter, the standard and most updated coiling techniques will be explained including advantages and drawbacks. The flow diverters will not be mentioned here.

\subsubsection{Antiplatelet Therapy}

Antiplatelet agents (aspirin and/or clopidogrel) should be given to the patients with unruptured aneurysms, 1 or 2 weeks before the treatment, to

\footnotetext{
N. Kuwayama

Division of Neuroendovascular Therapy, Department of Neurosurgery, University of Toyama,

Toyama, Japan

e-mail: kuwayama@med.u-toyama.ac.jp
}

prevent thromboembolic complications. When using a simple technique, a single agent (aspirin or clopidogrel) will be enough, while when using the adjunctive techniques, dual antiplatelet therapy (DAPT) will be recommended. If using the stents, DAPT is mandatory and should be kept within several months after the treatment. However, we should be aware of the hemorrhagic complications due to the DAPT medication (Match Study) [2]. In cases with ruptured aneurysms, single agent will be given after procedure or during treatment through the nasogastric tube. When using stents, loading dose of aspirin (300 mg) and clopidogrel (300-600 mg) should be given during or immediately after procedure. Increase of blood aspirin level is rapid but that of clopidogrel is slow, taking several hours even if the loading dose is given.

"VerifyNow (Accumetrics)" is an easy, instant, and portable instrument to assess the platelet aggregation activity of the patient immediately before treatment. The degree of suppression of platelet activity with either aspirin (ARU) or clopidogrel (PRU) can be monitored.

\subsubsection{Anesthesia}

Usually general anesthesia is favorable and selected to get immobilization of the patient particularly in case of ruptured aneurysms. If a patient would bleed in the procedure, vital sign 
could be controlled much better under intubated condition. Local anesthesia could be selected if the patient could well understand and stay still, but not recommended.

\subsubsection{Heparin}

Usually femoral arteries are selected for the access routes. After puncture of the artery, heparin should be given to maintain the ACT (activating clotting time) at 1.5-2 times of pretreatment level. We usually give 30005000 units in bolus intravenously at first and then 1000 units every $1 \mathrm{~h}$.

\subsubsection{Procedures of Coiling}

Though the simple technique should be the best, we have to utilize many kinds of adjunctive techniques to protect the parent artery from coil herniation out of the aneurysm dome. The practical techniques with microguidewires, microcatheters, balloons, and stents will be explained.

\subsection{Simple Coiling, Basic Techniques, and Follow-Up}

A 6Fr guiding catheter is usually selected to prepare for the second microcatheter usage. The first microcatheter is used for coiling and the second microcatheter (balloon catheter) is used for the adjuctive techniques. A microcatheter should be inserted into the aneurysm dome using a microguidewire. The detachable platinum coils are conceptionally divided into three types: framing, filling, and finishing coils. The decision to choose the size of framing coil should be same with or $0.5-1 \mathrm{~mm}$ smaller than the aneurysm diameter. When the aneurysm is spherical shaped, the size of framing coil should be the medium diameter between the long and short axes. The filling coils, shorter and smaller, come next and pack inside the framing coil. Then a few finishing coils follow and finalize the treatment. The finishing coil should be much softer and shorter. Final stage, the microguidewiere should be proceeded into the tip of the microcatheter and pulled back together securing the inside coils not coming with the catheter. The optimum packing rate will be $20-25 \%$ or more, and it is reported that the higher the packing rate, the less the recurrence rate [3].

The follow-up should be done under the monitor of plain craniogram and MRI time of flight (TOF) sequence. Morphological changes of the coil in the craniogram and/or inside flow into the aneurysm dome in MRI-TOF images will indicate the recurrence of the aneurysms. Then the angiogram should be done to evaluate the necessity of retreatment.

\subsection{Adjunctive Techniques}

A 6 or 7 Fr guiding catheter is usually selected to go with two or three catheters in combination. Sometimes a balloon-guiding catheter ( 7 or $8 \mathrm{Fr}$ ) is used to stabilize the intracranial remodeling balloon catheter or to control intraoperative bleeding. Here, five kinds of adjunctive techniques will be shown.

\subsection{1 \# Wire Protection Method}

(Fig. 22.1)

This is suitable to a small aneurysm arising from a small parent artery. Place a microguidewire passing across the aneurysm neck in the parent artery. It is sometimes useful to prevent the coil from protruding out of the aneurysm neck.

Figure 22.1 shows the anterior communicating artery (AcomA) aneurysm. The microguidewire is coming through the left internal carotid artery (ICA) and going to the distal right A2 segment through the AcomA, while the microcatheter is coming from the right ICA and inserted into the aneurysm dome. The coils do not come out of the dome with the protection of the microguidewire across the aneurysm neck. 

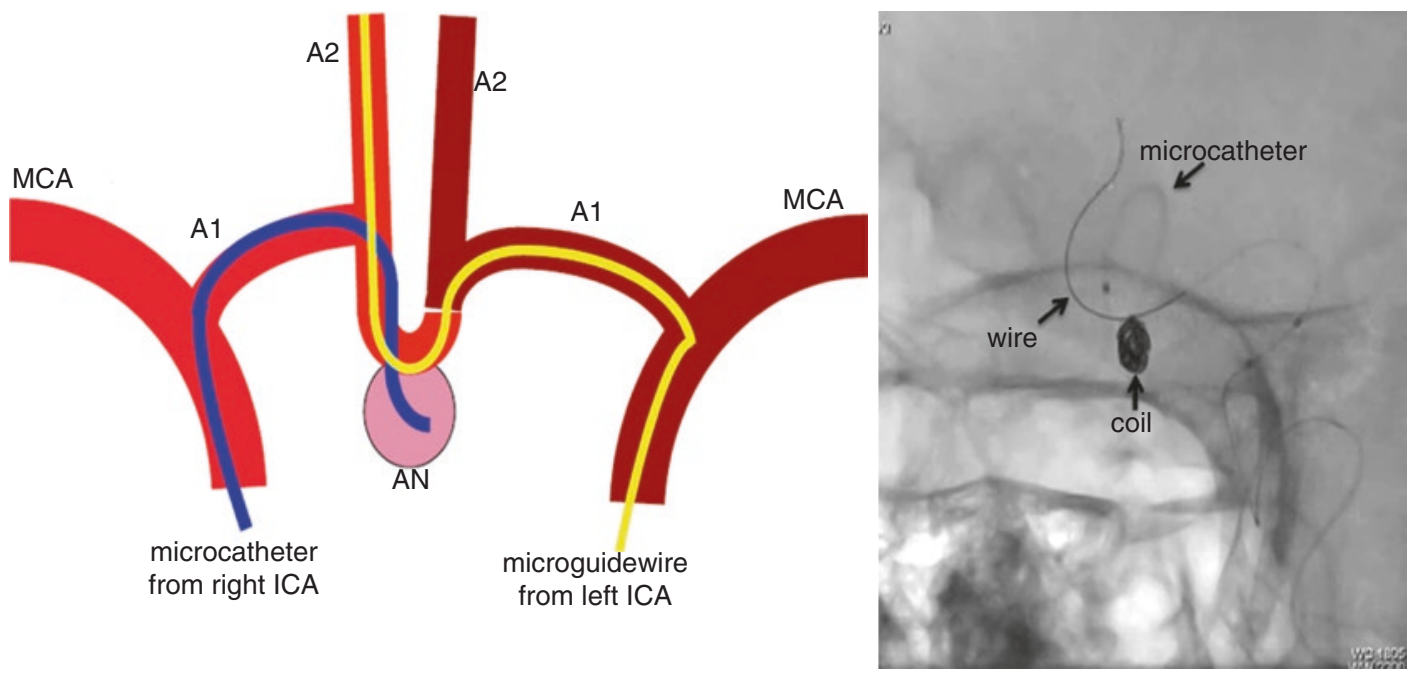

Fig. 22.1 The wire-assisted technique for an aneurysm of the anterior communicating artery

\subsection{2 \# Catheter Protection Method} (Fig. 22.2)

This is a modified method of the wire protection procedure. Place a microcatheter passing across the aneurysm neck in the parent artery. This is available in the small vessel like anterior cerebral arteries and in the posterior circulation.

Figure 22.2 demonstrates the aneurysm of the left VA-PICA. The microcatheter is coming from the contralateral right vertebral artery and going to the left PICA into its distal segment, while the second microcatheter is coming from the left vertebral artery and going into the aneurysm dome.

\subsection{3 \# Double-Catheter Method} (Fig. 22.3)

In case with wide-neck aneurysms, coils are easily herniated from the dome. Sometimes a loop of a detached framing coil may come out of the dome, being pushed by filling coils. In these cases, the double-catheter technique is often useful. First, send the two microcatheters into the aneurysm dome. Then start coiling. When the first coil makes a good frame in the dome, keep the coil undetached and push and hold the cathe- ter slightly pressing the framing coil to the dome side. Then start filling inside the frame with the second catheter. Usually after detaching two or three filling coils inside the dome, the framing coil will be stabilized well and not be herniated to the parent artery. Then the framing coil can be detached. Afterward, both the first and the second catheters can be used for the coil insertion depending on the situation.

Figure 22.3 shows the recurred aneurysm at the right posterior communicating artery (PcomA) after clipping. The previous multiple clips caused the tight stenosis of the ICA proximal to the aneurysm, allowing just one microcatheter to pass through the stenosis. While placing a microcatheter from the right ICA, the second microcatheter was coming from the contralateral left ICA through the AcomA, going down to the right ICA, and finally placed in the aneurysm dome. Thus, the wide-neck aneurysm was completely packed with the double-catheter technique.

\subsection{4 \# Balloon Remodeling Method} (Fig. 22.4)

First, send the balloon catheter to the distal parent artery and then send the microcatheter into 


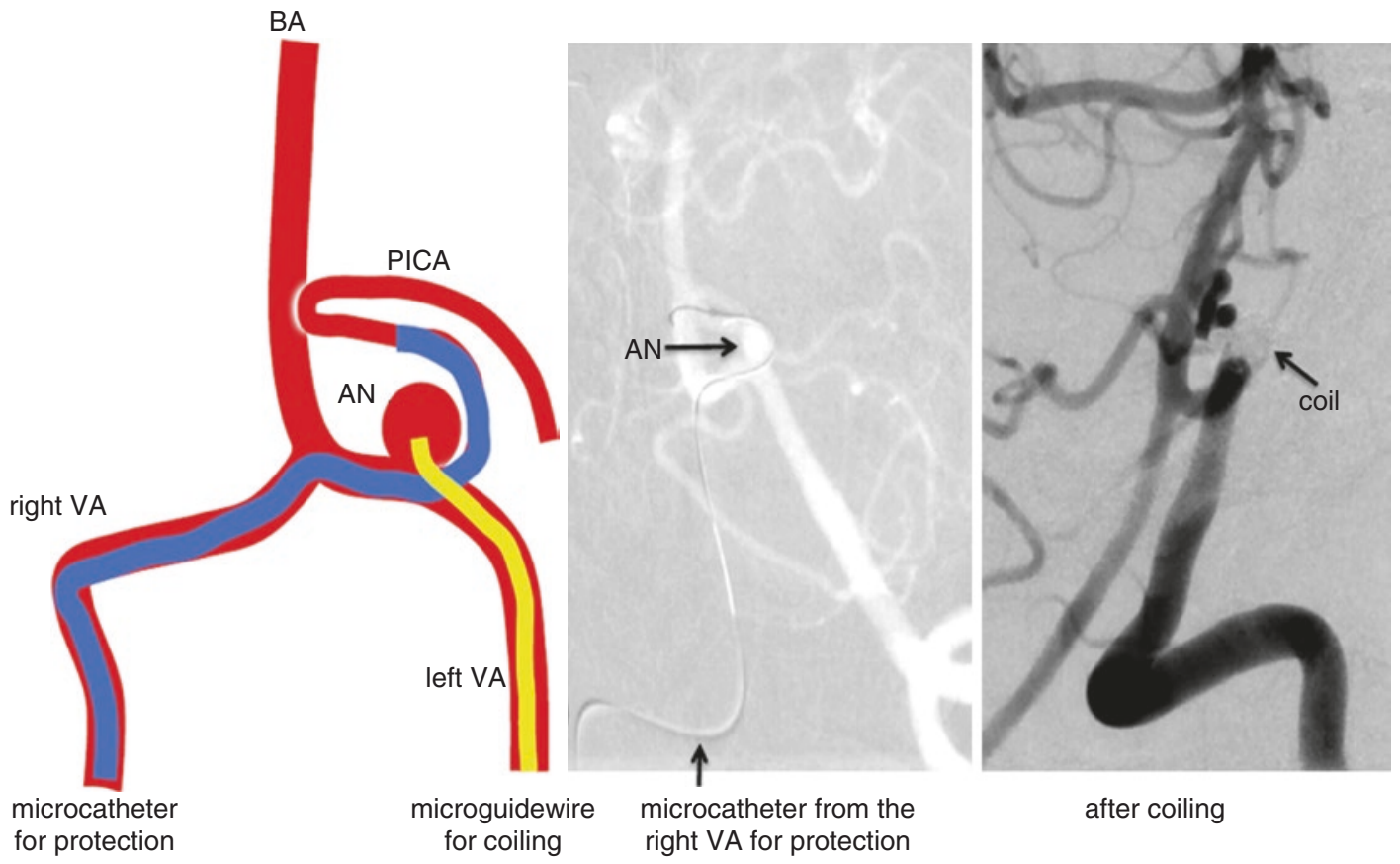

Fig. 22.2 The catheter-assisted technique for an aneurysm of the left vertebral artery (PICA)

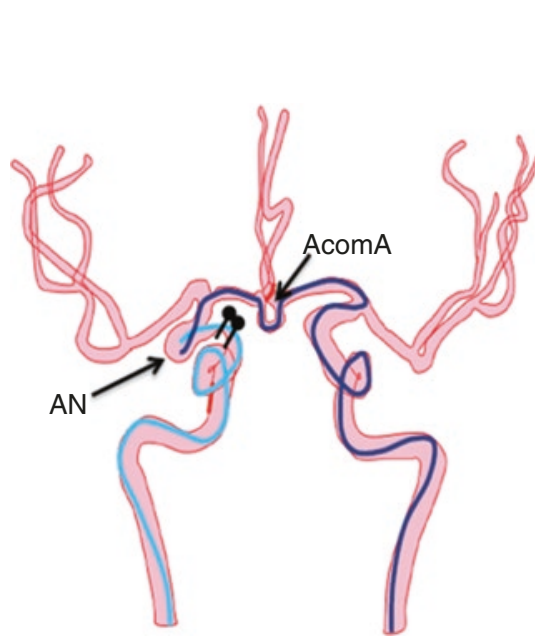

Schematic view of the double catheter techique from the bilateral ICA for an recurrent aneurysm of the right ICA after clipping. The previous clips make the tight ICA stenosis and only one catheter can pass through the ICA. The 2nd catheter is navigated from the left ICA throught AcomA.

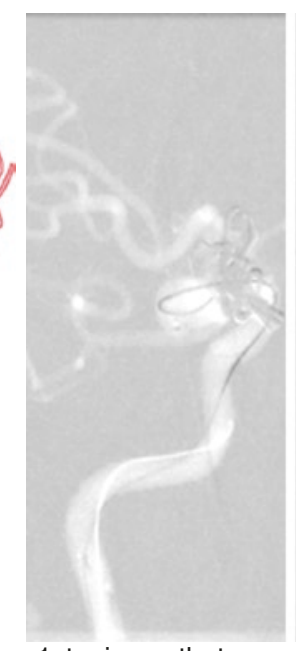

1st microcatheter from the right ICA

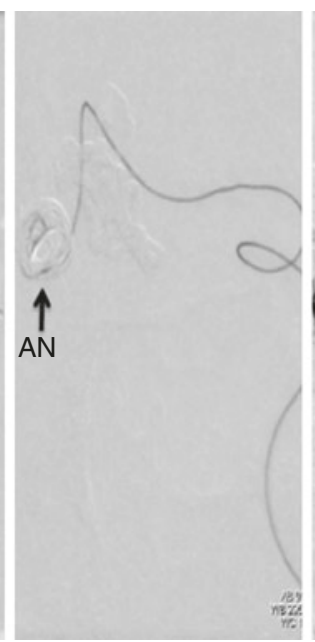

2nd microcatheter from the left ICA

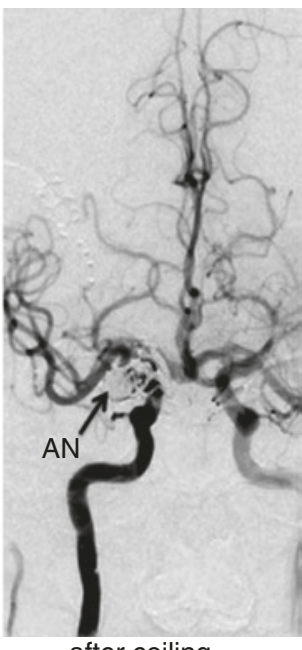

after coiling

Left: 1st microcatheter going from the right ICA to the aneurysm dome. Middle: 2nd microcatheter going from contralateral ICA to the aneurysm through the AcomA. Right: abgiogram after coiling.

Fig. 22.3 The double-catheter technique for a recurrent wide-neck aneurysm of the right ICA after clipping 

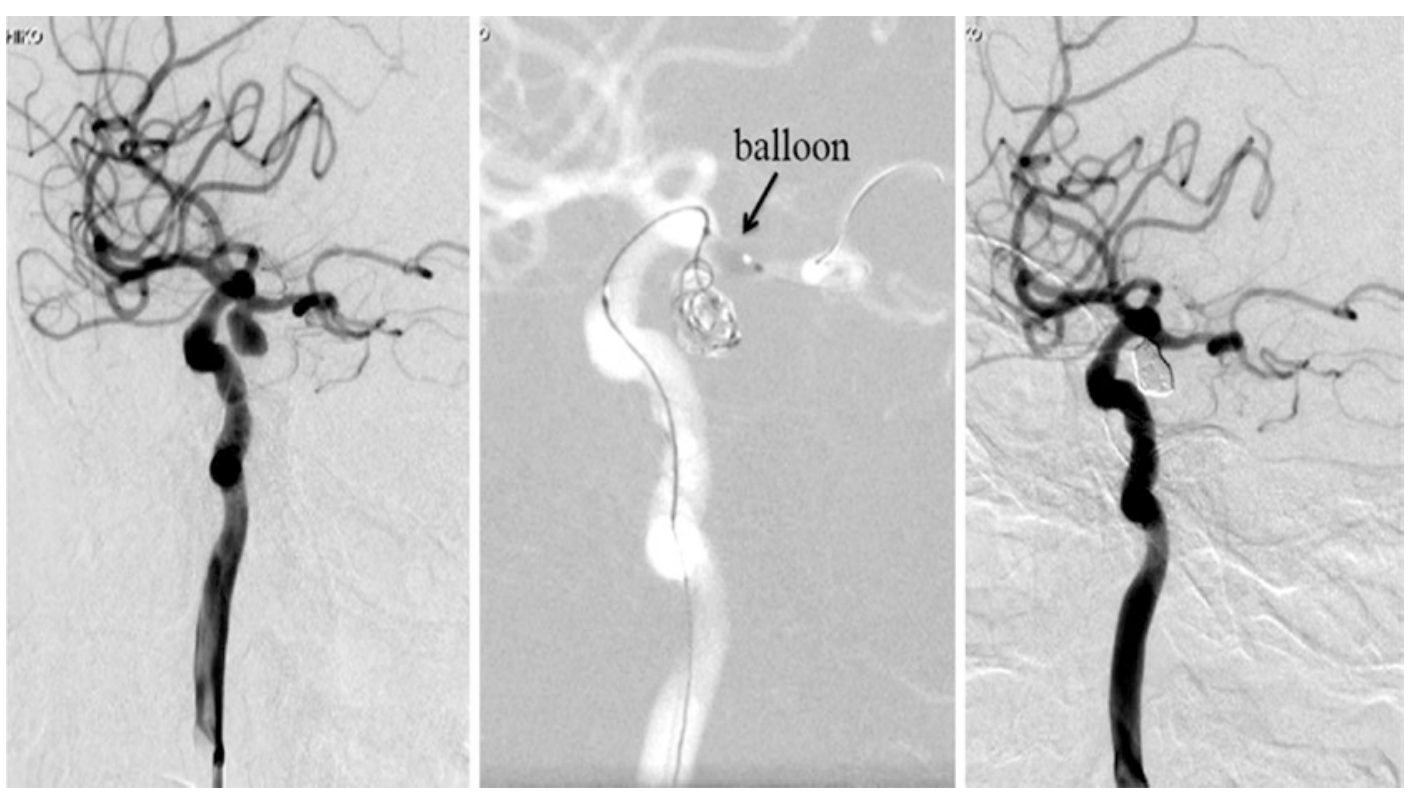

Fig. 22.4 The balloon remodeling technique for an aneurysm of the posterior communicating artery (PcomA). The aneurysm arises from the PcomA. This is the socalled pure Pcom aneurysm (left). The aneurysm is being packed with balloon remodeling technique (middle). The balloon is inflated in the parent PcomA to prevent coil herniation. The aneurysm is completely obliterated with coils keeping the PcomA patent (right) the aneurysm dome. The movement of one catheter will affect the position of the other catheter by the friction of both catheters. Surgeon should always be aware of the position of the tip of coil catheter, when moving the microballoon catheter, otherwise it may cause perforation of the aneurysm. When the balloon and microcatheter are placed properly, start coiling. The balloon can be inflated, if necessary, to prevent the coil coming out of the dome. Surgeon must remember that the inflated balloon will fix the tip of the coil catheter and prevent its free movement, causing rupture of the aneurysm. The balloon should be inflated under road mapping up to the size of the parent artery, not to cause the vessel rupture.

Figure 22.4 shows the so-called pure PcomA aneurysm (arising from PcomA itself). In the final stage of the procedure, the balloon was inflated in the PcomA preventing the finishing coils from herniating to the parent PcomA.

\subsection{5 \# Stent-Assisted Technique} (Fig. 22.5)

There are several kinds of remodeling stents: open cell type, closed cell type, braided type, etc. To use the stents, DAPT medication before procedure is mandatory to prevent thromboembolic complication. There are two ways to send the coil catheter to the aneurysm: one is jailing technique and the other is trans-cell technique. In case of jailing technique, send the stent catheter to the distal parent artery. Before opening the stent, send the coil catheter into the aneurysm dome. Then open and deploy the stent, and start coiling. In case of trans-cell technique, send the stent catheter to the distal parent artery and then open and deploy the stent. Send the coil catheter to the aneurysm through the cell of the stent, and start coiling. The demerit of the trans-cell method is to have a potential risk of wire or catheter perforation in cases with small aneurysms. 

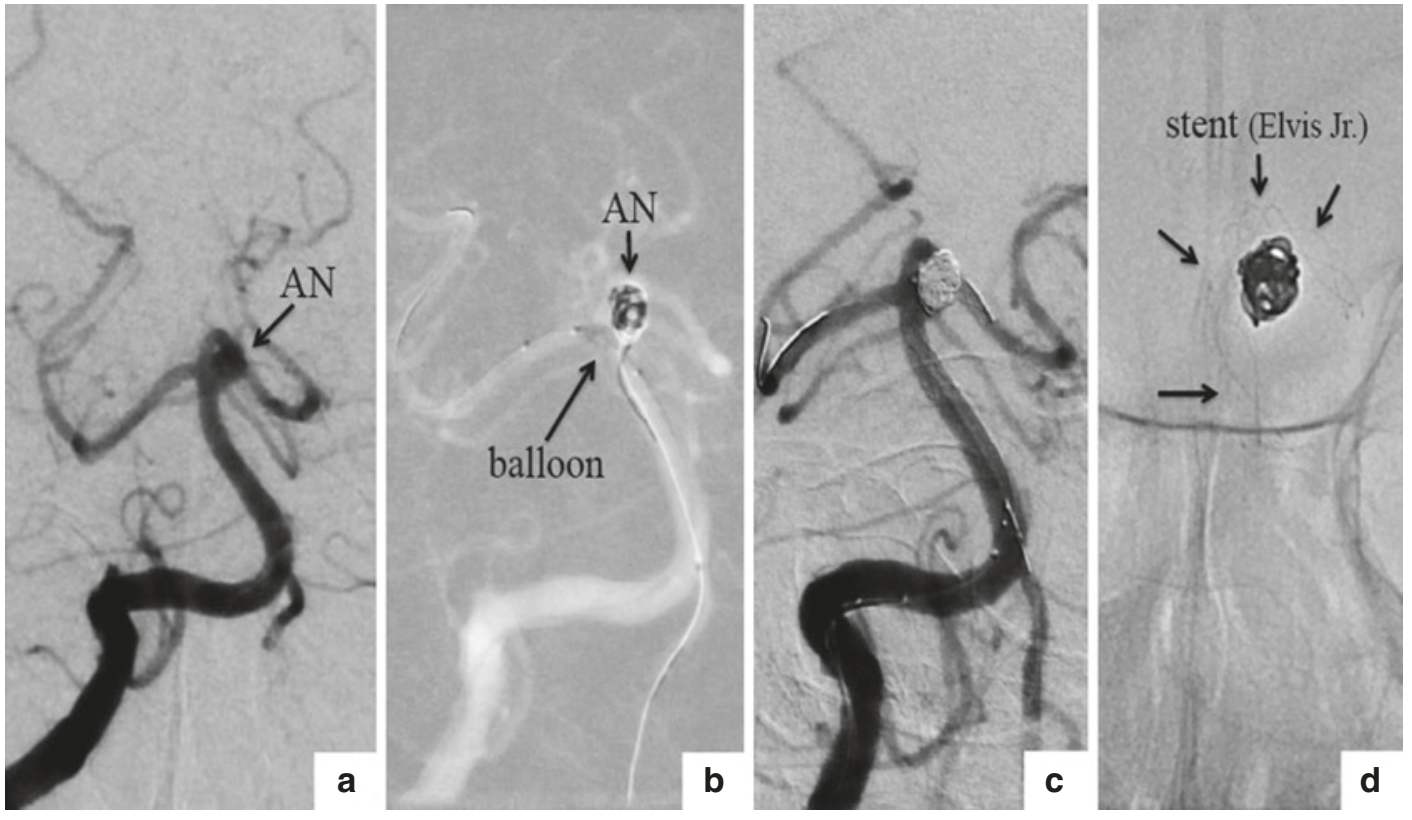

Fig. 22.5 The stent-assisted technique for an aneurysm of the origin of the superior cerebellar artery. (a) The aneurysm arises from the origin of the superior cerebellar artery (BA-SCA AN) (b) The aneurysm is being packed with balloon-assisted technique at first. The balloon is

At present, the jailing method seems to be dominantly used. The demerit of the jailing method is to fix the coil catheter preventing the free movement of the tip of the microcatheter. Recently semi-jailing method is frequently used, keeping the stent half open at the neck to secure the free movement of the coil catheter. In other words, the semi-jailing method makes the neck of the aneurysm smaller, allowing free catheter movement and easy and safe coiling. It is reported that stent-assisted coiling has smaller rate of recurrence due to the hemodynamic effect to the aneurysm [4].

Figure 22.5 demonstrates a case with the aneurysm of the superior cerebellar artery treated with balloon- and stent-assisted technique. Coiling started with balloon-assisted technique; then finally the stent (Elvis Jr.) was deployed from the PCA-P1 segment to the basilar artery. inflated from the right PCA-P1 segment to the upper basilar artery. (c) The aneurysm is completely obliterated with balloon- and stent-assisted technique. (d) Note the stent (arrows) is placed from the left PCA-P1 segment to the upper basilar artery

\section{References}

1. Molyneux A, Kerr R, Stratton I, Sandercock P, Clarke M, Shrimpton J, Holman R, International Subarachnoid Aneurysm Trial (ISAT) Collaborative Group. International Subarachnoid Aneurysm Trial (ISAT) of neurosurgical clipping versus endovascular coiling in 2143 patients with ruptured intracranial aneurysms: a randomised trial. Lancet. 2002;360(9342):1267-74.

2. Diener HC, Bogousslavsky J, Brass LM, Cimminiello C, Csiba L, Kaste M, et al. MATCH investigators. Aspirin and clopidogrel compared with clopidogrel alone after recent ischaemic stroke or transient ischaemic attack in high-risk patients (MATCH): randomised, double-blind, placebo-controlled trial. Lancet. 2004;364(9431):331-7.

3. Ding YH, Dai D, Lewis DA, et al. Angiographic and histologic analysis of experimental aneurysms embolized with platinum coils, Matrix, and HydroCoil. AJNR Am J Neuroradiol. 2005;26:1757-63.

4. Yang H, Sun Y, Jiang Y, Lv X, Zhao Y, Li Y, Liu A. Comparison of stent-assisted coiling vs coiling alone in 563 intracranial aneurysms: safety and efficacy at a high-volume center. Neurosurgery. 2015;77(2):241-7. 
Open Access This chapter is licensed under the terms of the Creative Commons Attribution 4.0 International License (http://creativecommons.org/licenses/by/4.0/), which permits use, sharing, adaptation, distribution and reproduction in any medium or format, as long as you give appropriate credit to the original author(s) and the source, provide a link to the Creative Commons license and indicate if changes were made.

The images or other third party material in this chapter are included in the chapter's Creative Commons license, unless indicated otherwise in a credit line to the material. If material is not included in the chapter's Creative Commons license and your intended use is not permitted by statutory regulation or exceeds the permitted use, you will need to obtain permission directly from the copyright holder.

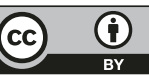

